\title{
THE HOMOTOPY ANALYSIS METHOD IN BIFURCATION ANALYSIS OF DELAY DIFFERENTIAL EQUATIONS
}

\author{
ANDREA BEL \\ Departamento de Matemática \\ Universidad Nacional del Sur \\ CONICET \\ Av. Alem 1254, 8000 Bahía Blanca, Argentina \\ andrea.bel@uns.edu.ar \\ WALTER REARTES \\ Departamento de Matemática \\ Universidad Nacional del Sur \\ Av. Alem 1254, 8000 Bahía Blanca, Argentina \\ reartes@uns.edu.ar
}

Received (to be inserted by publisher)

\begin{abstract}
In this paper we apply the homotopy analysis method (HAM) to study the van der Pol equation with a linear delayed feedback. The paper focuses on the calculation of periodic solutions and associated bifurcations, Hopf, double Hopf, Neimark-Sacker, etc. In particular we discuss the behavior of the systems in the neighborhoods of double Hopf points. We demonstrate the applicability of HAM to the analysis of bifurcation and stability.
\end{abstract}

Keywords: Homotopy analysis method - Delay differential equations - van der Pol equation Hopf bifurcation - Double Hopf bifurcation

\section{Introduction}

Dynamical systems modeled by nonlinear differential equations or nonlinear delay differential equations are difficult to solve by analytical methods. Delay differential equations are part of the domain of infinite dimensional dynamical systems. They are a special case of so-called functional differential equations [Hale \& Lunel, 1993]. This fact complicates specially the study of stability.

We can mention perturbative methods for solving these equations [Atay, 1998]. They require the existence of a parameter that takes small values. Since in the analysis of bifurcation and stability it is desirable to consider extensive regions of parameter space the usefulness of perturbative methods is restricted.

Among nonperturbative methods we find the homotopy perturbation method [He, 1999] and the homotopy analysis method (HAM) [Liao, 2004b]. The first one is a particular case of the latter. The HAM has been applied to solve ordinary and partial differential equations as well as delay differential equations [Abbasbandy, 2006; Kimiaeifar et al., 2009; Liao, 2004a,b,c, 2009; Liao \& Tan, 2007; Roohi et al., 2008; Sajid et al., 2007; Shakeri \& Dehghan, 2008].

In this work, we show that the HAM is an appropriate tool for studying nonlinear delay differential equations, focusing on bifurcation and stability analysis of the solutions. We take van der Pol's equation 
modified with a linear delayed feedback. Van der Pol-Duffing's equation has been studied applying HAM in [Kimiaeifar et al., 2009]. Atay [1998] studied van der Pol equation with delay. He showed the presence of Hopf bifurcation applying perturbative methods. Also Wei \& Jiang [2005] studied Hopf bifurcation in the equation of van der Pol with delayed feedback by means of normal forms and the center manifold theorem.

Our construction explores the parameter space and enable us to find all the periodic solutions with the HAM. Furthermore the high order for the cycle approximations allows us to apply a collocation method to study the stability in an efficient manner.

We consider van der Pol's equation with a linear delayed feedback

$$
x^{\prime \prime}(t)+\epsilon\left(x^{2}(t)-1\right) x^{\prime}(t)+x(t)=d \epsilon x(t-\tau),
$$

where $\epsilon$ and $d$ are positive real constants, and $\tau>0$ is the delay. We use the HAM [Liao, 2004b, 2009] to find analytical expressions for periodic solutions of the equation (1). By studying these solutions and their dynamics we find different types of bifurcations [Kuznetsov, 1995], some related to equilibrium, Hopf bifurcation, double Hopf bifurcation, etc., and others are bifurcations of the cycle itself, fold, and NeimarkSacker. The analysis of these bifurcations is essential in order to determine the behavior of the system when the parameters vary.

\section{Homotopy Analysis Method}

We suppose that there is a periodic solution of equation (1) with amplitude $a$ and frequency $\omega$. To find an analytic expression for it using the HAM it is suitable to normalize the equation. This is achieved by rescaling the variables $t$ and $x$ so that the amplitude and frequency of the periodic solution be 1 . However, we preserve $t$ and $x$ variable's names. The normalized equation is

$$
\omega^{2} x^{\prime \prime}(t)+\epsilon \omega\left(a^{2} x^{2}(t)-1\right) x^{\prime}(t)+x(t)=d \epsilon x(t-\omega \tau),
$$

where $\epsilon, d$ and $\tau \in \mathbb{R}^{+}$. We seek a periodic solution $x_{P}(t)$ of $(2)$ which verifies $x_{P}(0)=1$ and $x_{P}^{\prime}(0)=0$.

We consider the family of operators $\mathcal{H}_{q}$, that depends on a deformation parameter $q$

$$
\mathcal{H}_{q}[\phi]=(1-q) \mathcal{L}\left[\phi-x_{0}\right]-q h \mathcal{N}_{q}[\phi] .
$$

Here, $h \neq 0$ is a convergence control parameter, $x_{0}(t)$ is an initial approximation of the solution that verifies $x_{0}(0)=1$ and $x_{0}^{\prime}(0)=0, \mathcal{L}$ is the linear operator

$$
\mathcal{L}[\psi(t, q)]=\frac{\partial^{2} \psi(t, q)}{\partial t^{2}}+\psi(t, q),
$$

and $\mathcal{N}_{q}$ is an operator related to equation $(2) . \mathcal{N}_{q}$ is defined as

$$
\mathcal{N}_{q}[\phi(t, q)]=\Omega^{2}(q) \frac{\partial^{2} \phi(t, q)}{\partial t^{2}}+\epsilon \Omega(q)\left(A^{2}(q) \phi^{2}(t, q)-1\right) \frac{\partial \phi(t, q)}{\partial t}+\phi(t, q)-d \epsilon \phi(t-\Omega(q) \tau, q) .
$$

The aim of the procedure that we are developing is to find analytic functions (in the variable $q$ ) $\phi, A$ and $\Omega$ that verify $\mathcal{H}_{q}[\phi]=0$ for $q \in[0,1]$.

For $q=0$ and $q=1$ we have

$$
\mathcal{H}_{0}[\phi]=\mathcal{L}\left[\phi(t, 0)-x_{0}\right], \quad \text { and } \quad \mathcal{H}_{1}[\phi]=-h \mathcal{N}_{1}[\phi(t, 1)] .
$$

Note that $\phi(t, 0)-x_{0}(t)$ is a solution of $\mathcal{L}[\psi]=0$, then $\phi(t, 0)-x_{0}(t)=c_{1} \cos (t)+c_{2} \sin (t)$, with $c_{1}, c_{2} \in \mathbb{R}$. In addition, $\phi(t, 1)$ corresponds to the sought periodic solution, being $\Omega(1)$ and $A(1)$ its frequency and amplitude, respectively.

The conditions that must verify the solution of equation (2) imply that $\phi(0,1)=1$ and $\left.\frac{\partial \phi(t, 1)}{\partial t}\right|_{t=0}=0$, then we take

$$
\phi(0, q)=1,\left.\quad \frac{\partial \phi(t, q)}{\partial t}\right|_{t=0}=0,
$$

for all $q \in[0,1]$. These conditions together with those imposed on $x_{0}$ imply that $\phi(t, 0)=x_{0}(t)$. 
If $\phi, \Omega$ and $A$ are analytic functions in $q \in[0,1]$, there exists the following series

$$
\begin{aligned}
\phi(t, q) & =\sum_{k=0}^{+\infty} x_{k}(t) q^{k}, \\
\Omega(q) & =\sum_{k=0}^{+\infty} \omega_{k} q^{k}, \\
A(q) & =\sum_{k=0}^{+\infty} a_{k} q^{k} .
\end{aligned}
$$

Then $x_{P}(t)=\phi(t, 1), \omega=\Omega(1)$ and $a=A(1)$.

Series (4) depend on the $h$ parameter, they converge if it is possible to set an appropriate $h$ value.

The $k$-th term, $k \geq 1$, of these series is obtained by the next equation

$$
\mathcal{L}\left[x_{k}(t)-\left(1-\delta_{1 k}\right) x_{k-1}(t)\right]=\left.h \frac{1}{(k-1) !} \frac{\partial^{k-1} \mathcal{N}_{q}[\phi]}{\partial q^{k-1}}\right|_{q=0},
$$

and the initial conditions $x_{k}(0)=0, x_{k}^{\prime}(0)=0$. The above equation is obtained by the evaluation in $q=0$ of the $k$-th derivative with respect to $q$ of (3) previous to the replacement in it of the expressions (4) [Liao, 2009]. The problem in (5) is solved by the explicit inversion of the $\mathcal{L}$ operator.

The initial approximation $x_{0}(t)$ and each $x_{k}(t)$ term in the series of $x(t)$ must be periodic of integer period since we concentrate in the search of periodic solutions. Therefore the resolution of (5) requires that the non-periodic terms $t \cos (t)$ or $t \sin (t)$ become zero. This will be achieved by setting adequate values $\omega_{k-1}$ and $a_{k-1}$ in each step $k$, thus we can build the series expansions for $\omega$ and $a$.

In our case, we take $x_{0}(t)=\cos (t)$ because of the conditions for the solution of $(2)$. Then the right side of equation (5) (divided by $h$ ) for $k=1$ is

$$
\left(1-\omega_{0}^{2}-d \epsilon \cos \left(\omega_{0} \tau\right)\right) \cos (t)+\epsilon \omega_{0}\left(1-\frac{a_{0}^{2}}{4}-\frac{d}{\omega_{0}} \sin \left(\omega_{0} \tau\right)\right) \sin (t)-\epsilon \omega_{0} \frac{a_{0}^{2}}{4} \sin (3 t),
$$

hence $\omega_{0}$ and $a_{0}$ must be the solution of the system

$$
\left\{\begin{array}{l}
1-\omega_{0}^{2}-d \epsilon \cos \left(\omega_{0} \tau\right)=0 \\
1-\frac{a_{0}^{2}}{4}-\frac{d}{\omega_{0}} \sin \left(\omega_{0} \tau\right)=0
\end{array}\right.
$$

We point out that the first equation in (6) can be solved graphically for $\omega_{0}$. Consequently, we can solve the second equation for $a_{0}$. The number of solutions of these equations gives a first estimation of the number of cycles for each value of the parameters. At the end of this section we display how this estimation can be improved.

For $k \geq 1$ the conditions for $\omega_{k}$ and $a_{k}$ are linear and the system always has solution.

We can calculate $x_{1}(t)$, once the values of $\omega_{0}$ and $a_{0}$ have been determined. Then we write the equation for $x_{2}(t)$, from which we get a linear system, it allows us to determine $\omega_{1}$ and $a_{1}$. In this way we can obtain analytical expressions of the periodic solution, frequency and amplitude up to arbitrary order in $q$ as a function of $h$. All these calculations can be easily made with Mathematica.

It is time to determine an appropriate value for $h$. The expressions obtained for $\omega, a$ and $x(t)$ are polynomials in $h$. As shown in the book of Liao [2004b], the observation of the behavior of these polynomials allows us to select an appropriate value for $h$. For values of this parameter for which the series is convergent, such polynomials converge to a value that is independent of $h$, as the order tends to infinity. Thus, the polynomial plots give us a rough idea of the place where these intervals are found and therefore we select an appropriate value for $h$. Fig. 1 a) shows several of these polynomials where the intervals are clearly distinguishable. Specifically, in an expression of order 20 for a computed cycle with $\epsilon=0.5, \tau=12$, and $d=1.35$. A remarkable coincidence is shown in Fig. $1 \mathrm{~b}$ ). Therein, the cycle is plotted as a function of $t$ for $h=-0.6$ together with a numerical solution computed by Mathematica. This fact is observed in all the calculations made in this work. The detailed study of these questions is out of the scope of this paper. 
a)

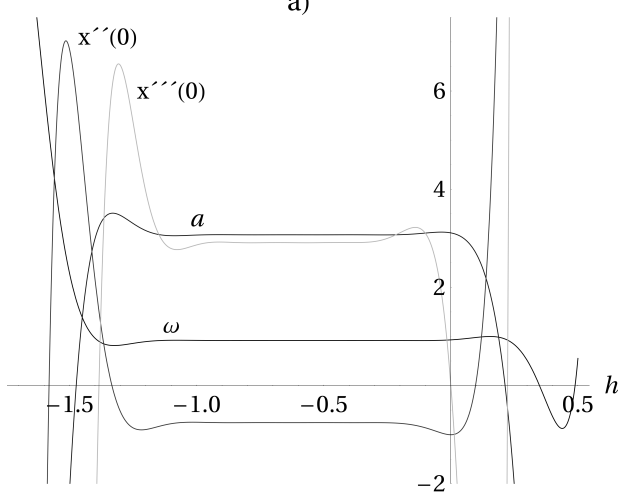

b)

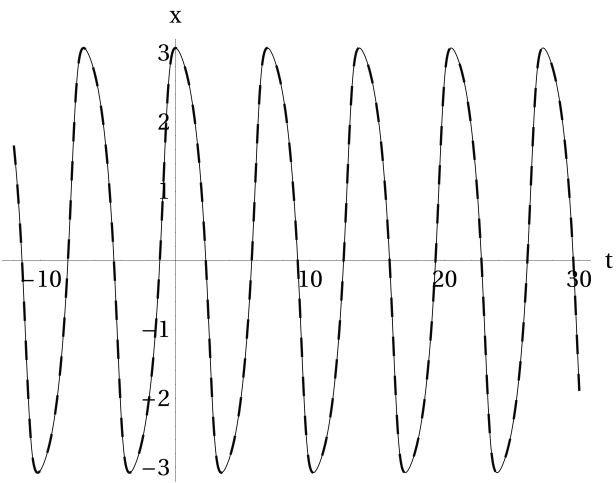

Fig. 1. a) Polynomials as function of $h$; b) - - -: cycle HAM of order 20; - : cycle numerical approximation.

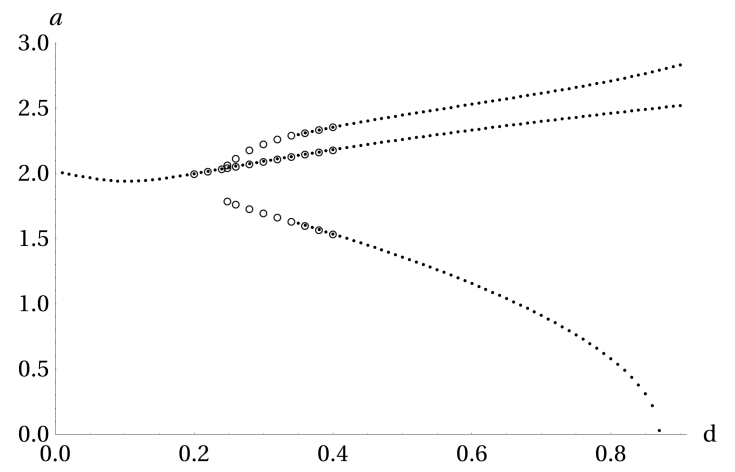

Fig. 2. Amplitude of cycles for $\epsilon=1.4142$ and $\tau=9 . \cdots$ : without iteration of initial conditions; $\circ \circ \circ$ : with three iterations of initial conditions.

\subsection{Iterated $H A M$}

As it is usual with the optimal solution search methods, the use of iterations greatly improves our HAM results. Iterations are used in two ways, one related to calculation of initial conditions and other for particular cycle calculation.

In some regions of the parameter space (specially if $\tau$ takes large values), the computation of the cycles was incomplete when (6) renders the initial conditions. Therefore it is necessary to correct the generation of these initial conditions.

Suppose that the values of the parameters are fixed. It is possible to generate new initial conditions by means of iterations of the initial solution $x_{0}(t)$. Each iteration is based on three steps: 1$)$ to solve the HAM with order one, 2) to generate a system with the obtained function (in the same way that we obtain (6)) and to calculate new initial conditions, which are at least the same amount as in the previous step, and 3) to use the obtained function as input for the next iteration. The stopping criterion for this generation process is that the number of initial conditions does not vary after a predetermined number of times.

The new process of the generation of the initial conditions is illustrated in Fig. 2. It shows the amplitude of the cycles for $\epsilon=1.4142$ and $\tau=9$, as a function of $d$, with three iterations (unfilled dots) and without iterations (dots). It is clear that only one of the cycles is detected for $d$ between 0.2455 and 0.34 .

Once the initial conditions are determined we apply the for us named iterated HAM. It fit in the metaheuristics search, and allows us to explore the space of $2 \pi$ periodic functions of unitary amplitude by transforming iteratively the solutions.

The iterated HAM consist of the calculation a fixed number of terms in the expansion of the solution, to fix an appropriate value of $h$ and use the obtained function as input to the next iteration. The process ends when the difference between two successive iterations is less than certain tolerance value. 
By setting the parameter $h$, the number of terms involved in the calculation is greatly reduced. Thus the advantage of the iterated HAM is the improvement in the speed of calculations of the cycles. Moreover, the process does not affect the quality of the solution as an approximation of the exact. In fact, it generally provides better approximations than those obtained with HAM.

For example, we consider $\epsilon=0.5, \tau=12$ and $d=0.35$. These are the values for the cycle of Figure 1 . Table 1 contains the values of $\omega, a, x^{\prime \prime}(0)$, and $x^{\prime \prime \prime}(0)$ for this cycle. They were calculated with HAM and iterated HAM, taking different orders and number of iterations, respectively. The order for the iterated HAM is 5 in all cases. We observe the similarity between the values obtained with HAM of order 40 and iterated HAM with 8 iterations, however the calculation time of the latter was close to a $3 \%$ of the first one.

Table 1. Values of $\omega, a, x^{\prime \prime}(0)$, and $x^{\prime \prime \prime}(0)$ for the cycle $\epsilon=0.5, \tau=12$, and $d=1.35$.

\begin{tabular}{lcccccc}
\hline & \multicolumn{3}{c}{ HAM without iterations } & \multicolumn{3}{c}{ HAM order 5 with iterations } \\
& order 10 & order 20 & order 40 & 2 iter & 4 iter & 8 iter \\
\hline & \multirow{2}{*}{0.920582} & 0.920284 & 0.920325 & 0.920219 & 0.920322 & 0.920324 \\
$\omega$ & 3.070616 & 3.072865 & 3.072773 & 3.073421 & 3.072783 & 3.072775 \\
$x^{\prime \prime}(0)$ & -0.753920 & -0.753933 & -0.753726 & -0.753705 & -0.753706 & -0.753726 \\
$x^{\prime \prime \prime}(0)$ & 2.892260 & 2.911034 & 2.912385 & 2.943078 & 2.912267 & 2.912386 \\
\hline
\end{tabular}

\section{Stability of Equilibrium and Hopf Bifurcation}

By the substitution of $x(t)$ by a constant $x^{*}$ in equation (2) we obtain $x^{*}=d \epsilon x^{*}$. Then, if $d \epsilon \neq 1, x=0$ is the unique equilibrium. Otherwise there are infinite non-isolated equilibria. The stability of $x=0$ is determined using the characteristic equation

$$
\lambda^{2}-\epsilon \lambda+1-d \epsilon e^{-\tau \lambda}=0 .
$$

Thus, the possible changes in stability are related to the existence of zero real roots or pure imaginary conjugate complex roots. The characteristic equation have the root $\lambda=0$ only if $d \epsilon=1$. For the existence of pure imaginary roots the condition obtained by replacing $\lambda=i \omega$ in (7) is

$$
-\omega^{2}-i \omega \epsilon+1-d \epsilon e^{-i \omega \tau}=0 .
$$

The real and imaginary part of (8) are

$$
\left\{\begin{array}{l}
1-\omega^{2}-d \epsilon \cos (\omega \tau)=0 \\
-\omega \epsilon+d \epsilon \sin (\omega \tau)=0
\end{array}\right.
$$

This system defines a surface $S$ in the parameter space $(d, \tau, \epsilon)$ (see Fig. 3), and it is equivalent to that obtained by setting $a_{0}=0$ in (6). On the surface may be a change of stability due to a Hopf bifurcation. Furthermore, in the self-intersections of $S$, the existence of two pairs of conjugate complex roots is an indication of the possible existence of double Hopf bifurcations. In Fig. 4 we see Hopf bifurcation curves for the three fixed values of $\epsilon$.

From equations (9) we obtain expressions for $\tau$ and $d$ as a functions of $\omega$ and the parameter $\epsilon$,

$$
\begin{aligned}
& d=\frac{\sqrt{1+\left(\epsilon^{2}-2\right) \omega^{2}+\omega^{4}}}{\epsilon} \\
& \tau=\frac{1}{\omega}\left(2 k \pi+\arctan \left(\frac{\epsilon \omega}{1-\omega^{2}}\right)\right) .
\end{aligned}
$$

Here $k \in \mathbb{N} \cup\{0\}$ and the arctan function is defined so that it is continuous in $\omega=1$. For each value of $k$ these expressions determine a sheet of the surface $S$. On the other hand, by squaring equations (9) and by 


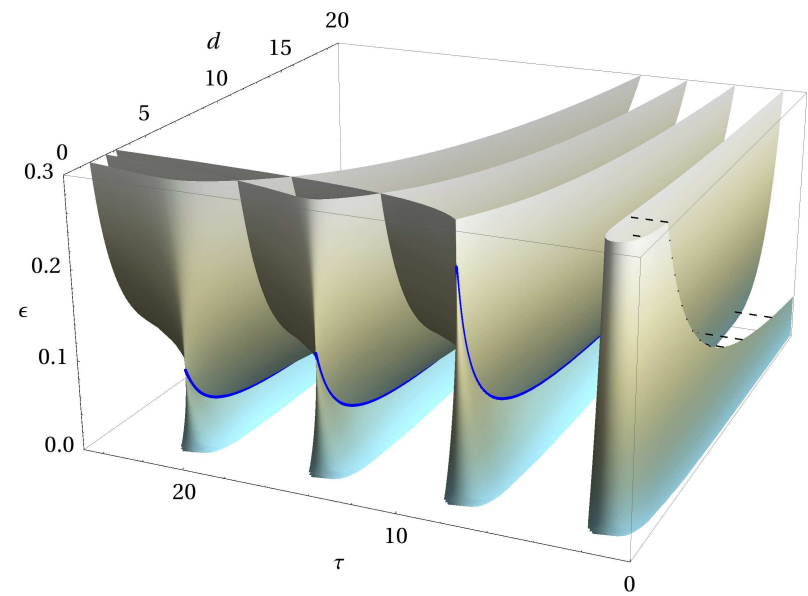

Fig. 3. Surface $S$ in the space $(d, \tau, \epsilon)$. - : curves of self-intersections; - - -: segments where $d \epsilon=1$.
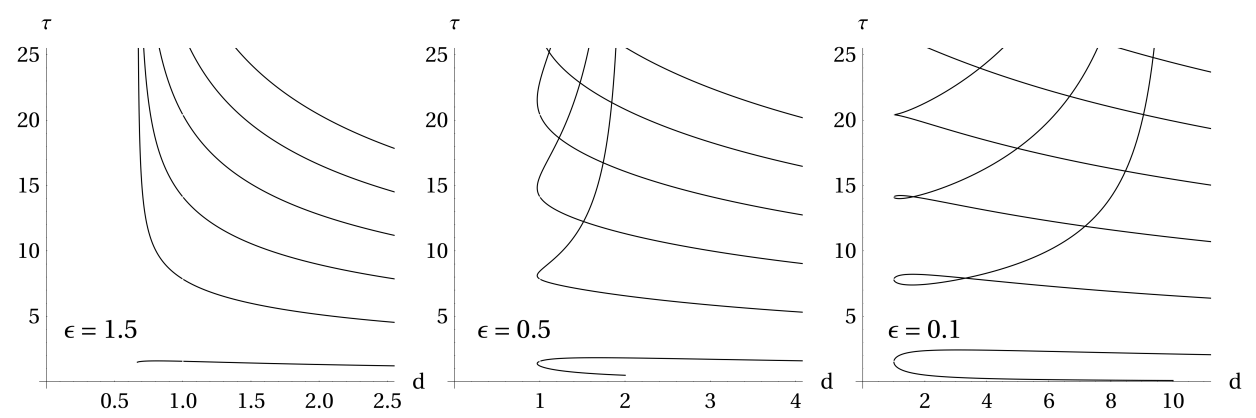

Fig. 4. Hopf bifurcation curves for three values of $\epsilon$.

performing some simple operations we obtain

$$
\omega_{1,2}=\sqrt{\frac{2-\epsilon^{2} \pm \epsilon \sqrt{\epsilon^{2}-4\left(1-d^{2}\right)}}{2}} .
$$

The self-intersections of the surface determine the necessary conditions for the existence of double Hopf bifurcations. The surface has a self-intersection when

$$
\left\{\begin{array}{l}
\tau\left(\omega_{1}\right)=\tau\left(\omega_{2}\right), \\
d\left(\omega_{1}\right)=d\left(\omega_{2}\right) .
\end{array}\right.
$$

The second condition implies

$$
\omega_{1}^{2}+\omega_{2}^{2}=2-\epsilon^{2}
$$

hence there are intersections only if $\epsilon<\sqrt{2}$. On the other hand, the first condition is

$$
\omega_{2}\left(2 k_{1} \pi+\arctan \left(\frac{\epsilon \omega_{1}}{1-\omega_{1}^{2}}\right)\right)=\omega_{1}\left(2 k_{2} \pi+\arctan \left(\frac{\epsilon \omega_{2}}{1-\omega_{2}^{2}}\right)\right) .
$$

If $k_{1} \neq k_{2}$, the intersection is between different sheets of the surface. In relation with the stability of the equilibrium the most interesting case is that corresponding to intersections of the same sheet, i.e., $k_{1}=k_{2}$.

If the double Hopf bifurcation exists and $\omega_{2} / \omega_{1}=p / q$, with $p, q \in \mathbb{N}$ it is said that the bifurcation is resonant, otherwise it is called non-resonant. Next we obtain the conditions for resonant double Hopf bifurcation. From the equation (10) we have

$$
\omega_{1}=\sqrt{\frac{2-\epsilon^{2}}{1+\left(\frac{p}{q}\right)^{2}}},
$$


from (11) we obtain

$$
\frac{p}{q}\left(2 k_{1} \pi+\arctan \left(\frac{\epsilon \omega_{1}}{1-\omega_{1}^{2}}\right)\right)=2 k_{1} \pi+\arctan \left(\frac{p q \epsilon \omega_{1}}{q^{2}-p^{2} \omega_{1}^{2}}\right),
$$

for $k_{1} \in \mathbb{N} \cup\{0\}$ fixed. Once are giving the values to $p$ and $q$, according to the desired resonance, the previous equations define a system that allows us to find the $\epsilon$ parameter. If there is any in which the corresponding intersection verifies the resonance condition. If $k_{1}$ varies, in some cases, it is possible that more than one $\epsilon$ verifies the condition.

\section{Stability of Periodic Solutions}

To study the stability of a periodic solution $x_{P}(t)$ (with amplitude $a$ and frequency $\omega$ ) we write $x(t)=$ $x_{P}(t)+v(t)$. Its substitution in equation (2) brings forth

$$
\omega^{2} v^{\prime \prime}(t)+\epsilon \omega\left(a^{2}\left(x_{P}(t)+v(t)\right)^{2}-1\right) v^{\prime}(t)+\epsilon \omega\left(a^{2}\left(2 x_{P}(t)+v(t)\right) v(t)\right) x_{P}^{\prime}(t)+v(t)=d \epsilon v(t-\omega \tau) .
$$

If the equilibrium $v=0$ of $(12)$ is asymptotically stable then $x_{P}(t)$ is asymptotically stable, otherwise it is unstable. In order to determine the stability of the trivial solution the linearization of equation (12) gives

$$
\omega^{2} v^{\prime \prime}(t)+\epsilon \omega\left(a^{2} x_{P}^{2}(t)-1\right) v^{\prime}(t)+\left(2 \epsilon \omega a^{2} x_{P}(t) x_{P}^{\prime}(t)+1\right) v(t)=d \epsilon v(t-\omega \tau) .
$$

Equation (13) is a linear delay equation, with $2 \pi$ periodic coefficients, and it is a special case of equation

$$
\begin{gathered}
\dot{\mathbf{x}}=\mathbf{A}_{1}(t) \mathbf{x}(t)+\mathbf{A}_{2}(t) \mathbf{x}(t-\xi), \\
\mathbf{x}(t)=\varphi(t) \quad \text { if } \quad-\xi \leq t \leq 0,
\end{gathered}
$$

where $\mathbf{x}(t)$ is an $n$-dimensional vector of states, $\xi>0$ is the delay, $\varphi \in C([-\xi, 0])$, and $\mathbf{A}_{1}(t)$ and $\mathbf{A}_{2}(t)$ are $n \times n$ periodic matrices with period $T$, i.e. $T$ is the smallest positive real number such that $\mathbf{A}_{i}(t+T)=\mathbf{A}_{i}(t)$.

We can define a monodromy operator associated to equation (14) similarly to the case of time-periodic ODE's [Hale \& Lunel, 1993]. The fundamental difference is that it acts on an infinite dimensional space of functions, which are the initial conditions of the equation. This operator has an infinite number of eigenvalues (Floquet multipliers). It is verified that there is always a trivial eigenvalue 1, and that the solution $\mathbf{x}=0$ of the equation is asymptotically stable if the non-trivial eigenvalues have modulus less than 1, and it is unstable if any of them has magnitude greater than 1 [Hale \& Lunel, 1993]. One way to find these eigenvalues is to construct a finite dimensional operator which approximates the monodromy operator. In this paper we apply the Chebyshev collocation method [Butcher \& Mann, 2009].

\subsection{Chebyshev Collocation Method.}

Chebyshev polynomials of the first kind are defined for $n \geq 0$ by

$$
T_{n}(\cos (\theta))=\cos (n \theta) .
$$

The shifted Chebyshev polynomials are defined in the interval $[a, b]$ as follows

$$
T_{n}^{*}(s)=T_{n}\left(\frac{2 s-(a+b)}{b-a}\right) .
$$

These polynomials form an orthogonal set, i.e.

$$
\int_{a}^{b} T_{n}^{*}(s) T_{m}^{*}(s) w(s) d t=0, \quad \text { if } \quad n \neq m,
$$

where $w(s)=\left(s(a+b)-s^{2}-a b\right)^{-1 / 2}$.

The Chebyshev collocation points are points in the interval $[-1,1]$, defined as $t_{j}=\cos (j \pi / N)$, for $j=0,1, \ldots, N$. Let $m=N+1$ be the number of collocation points.

A spectral differentiation matrix for Chebyshev collocation points is obtained by interpolating a polynomial through the collocation points. It is follows by the differentiation and the evaluating of the resulting 
polynomial at the point of collocation [Bueler, 2004]. For any order $m$, the elements of the Chebyshev spectral differentiation matrix, $\mathbf{D} \in \mathbb{M}_{m}$, are defined as

$$
\begin{gathered}
D_{00}=\frac{2 N^{2}+1}{6}, \quad D_{N N}=-\frac{2 N^{2}+1}{6}, \\
D_{j j}=\frac{-t_{j}}{2\left(1-t_{j}^{2}\right)}, \quad j=1, \ldots, N-1, \\
D_{i j}=\frac{c_{i}(-1)^{i+j}}{c_{j}\left(t_{i}-t_{j}\right)}, \quad i \neq j, \quad i, j=0, \ldots, N,
\end{gathered}
$$

where $c_{i}=\left\{\begin{array}{c}2 \quad \text { if } \quad i=0, N \\ 1 \quad \text { if } \quad i \neq 0, i \neq N\end{array}\right.$.

Furthermore, if the equation is $n$-dimensional, we can define the differential operator $\mathbb{D}$ of order $n m \times n m$ as $\mathbb{D}=\mathbf{D} \otimes I_{n}$.

Chebyshev collocation method allows us to approximate the solution values of equation (14) at the collocation points of the solution interval, from the values of the initial condition at the collocation points, $s_{j}=(\cos (j \pi / N)-1) \xi / 2$, in the interval $[-\xi, 0]$.

First we consider the case $T=\xi$ analyzed in Butcher \& Mann [2009]. Let $\mathbf{m}_{1}$ be the set of $m$ values of the solution in the interval $[0, T]$ and $\mathbf{m}_{\varphi}$ the set of $m$ values of the initial function $\varphi(t)$ in $[-T, 0]$. Since these values are numbered from the right to the left the continuity condition is $\mathbf{m}_{1 N}=\mathbf{m}_{\varphi_{0}}$. Replacing $\mathbf{m}_{1}$ and $\mathbf{m}_{\varphi}$ in (14) begets

$$
\hat{\mathbf{D}} \mathbf{m}_{1}=\hat{\mathbf{M}}_{A_{1}} \mathbf{m}_{1}+\hat{\mathbf{M}}_{A_{2}} \mathbf{m}_{\varphi} \text {. }
$$

The matrix $\hat{\mathbf{D}}$ is obtained from $\mathbb{D}$ by replacing the last $n$ rows by $\left[\begin{array}{lllll}\mathbf{0}_{n} & \mathbf{0}_{n} & \ldots & \mathbf{I}_{n}\end{array}\right]$, to ensure the conditions of continuity. Here $\mathbf{0}_{n}$ and $\mathbf{I}_{n}$ are the $n \times n$ matrices of zeros and the identity, respectively. In addition, the obtained matrix should be multiplied by $2 / T$ due to the rescaling of the polynomials to the interval $[0, T]$. The remaining matrices in (15) are defined as

$$
\begin{aligned}
\hat{\mathbf{M}}_{A_{1}} & =\left[\begin{array}{ccccc}
\mathbf{A}_{1}\left(s_{0}\right) & & & & \\
& \mathbf{A}_{1}\left(s_{1}\right) & & & \\
& & \ddots & & \\
& & & \mathbf{A}_{1}\left(s_{N-1}\right) & \\
\mathbf{0}_{n} & \mathbf{0}_{n} & \ldots & \mathbf{0}_{n} & \mathbf{0}_{n}
\end{array}\right], \\
\hat{\mathbf{M}}_{A_{2}} & =\left[\begin{array}{ccccc}
\mathbf{A}_{2}\left(s_{0}\right) & & & & \\
& \mathbf{A}_{2}\left(s_{1}\right) & & & \\
& & \ddots & & \\
& & & \mathbf{A}_{2}\left(s_{N-1}\right) & \\
\mathbf{I}_{n} & \mathbf{0}_{n} & \ldots & \mathbf{0}_{n} & \mathbf{0}_{n}
\end{array}\right],
\end{aligned}
$$

where $\mathbf{A}_{i}\left(s_{j}\right)$ is the $n \times n$ matrix which results from evaluation of $\mathbf{A}_{i}$ in the $j$-th collocation point.

From the collocation method we define a finite dimensional monodromy matrix $\mathbf{U}$ whose eigenvalues approximate the Floquet multipliers [Butcher \& Mann, 2009]. It follows from the definition of the monodromy operator and since $T=\xi$, the definition of $\mathbf{U}$ as the mapping of the values of the solution in successive intervals, i.e. $\mathbf{m}_{1}=\mathbf{U m}_{\varphi}$, then (15) leads to

$$
\mathbf{U}=\left(\hat{\mathbf{D}}-\hat{\mathbf{M}}_{A_{1}}\right)^{-1} \hat{\mathbf{M}}_{A_{2}} \text {. }
$$

Now we can calculate $\mathrm{nm}$ eigenvalues. The accuracy of the obtained Floquet multipliers generally improves with the increase in the number of collocation points.

The dynamics study of the cycles requires the previous calculation to the general case $T \neq \xi$. Here, our implementation follows the idea proposed in Bueler [2004] for numerical approximation of eigenvalues. We do not make a detailed numerical analysis of this calculation. 


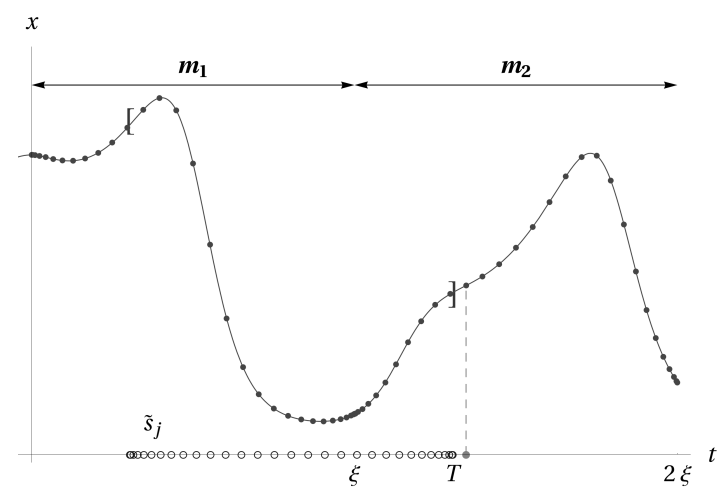

Fig. 5. Collocation points and the grid for interpolation.

We consider $0<\xi<T \leq 2 \xi$, because the remainder cases can be deduced from this. To obtain the solution in period $T$, with initial condition $\varphi$, it is necessary to calculate

$$
\begin{aligned}
& \mathbf{m}_{1}=\mathbf{U} \mathbf{m}_{\varphi} \quad \text { in }[0, \xi], \\
& \mathbf{m}_{2}=\mathbf{U m}_{1}=\mathbf{U}^{2} \mathbf{m}_{\varphi} \quad \text { in }[\xi, 2 \xi] \text {, }
\end{aligned}
$$

with the continuity conditions $\mathbf{m}_{2 N}=\mathbf{m}_{10}$. Then, we have expressions for the values of the solutions in $2 m$ collocation points, $m$ corresponding to the interval $[0, \xi]$ and $m$ to the interval $[\xi, 2 \xi]$.

$\mathbf{U}$ is not the monodromy operator because it must act between the spaces of continuous functions $C([-\xi, 0])$ and $C([T-\xi, T])$. It is necessary to adapt the definition and then to build a new operator $\tilde{\mathbf{U}}$, which transforms $\mathbf{m}_{\varphi}$ in $\tilde{\mathbf{m}}$, in order to represent the monodromy operator, and finally to approximate its eigenvalues.

Let $M \in \mathbb{N}$ be such that $2 \xi+s_{M} \leq T \leq 2 \xi+s_{M-1}$, where $s_{j}$ are the collocation points in the interval $[-\xi, 0]$. The values are numbered from the right to the left with starter at 0 . Consider the section of solution represented for the remaining $(N+1-(M-1))$ values of $\mathbf{m}_{2}$ given by $\left\{\mathbf{m}_{2(M-1)}, \ldots, \mathbf{m}_{2 N}\right\}$, and the first $M$ values of $\mathbf{m}_{1},\left\{\mathbf{m}_{10} \ldots, \mathbf{m}_{1(M-1)}\right\}$, an extra point in $\mathbf{m}_{1}$ is considered due to the continuity condition $\mathbf{m}_{2 N}=\mathbf{m}_{10}$. Fig. 5 shows an example of the situation, values in $\mathbf{m}_{1}$ and $\mathbf{m}_{2}$ are plotted in dots on the solution, and also we observe the point $2 \xi+s_{M-1}$ and its corresponding value $\mathbf{m}_{2(M-1)}$. In the same figure, the brackets limit the section of the solution that we want to approximate. On the horizontal axis we show the collocation points of the interval $[T-\xi, T]$, defined as $\tilde{s}_{j}=T+s_{j}$. In general the collocation point corresponding to the value $\mathbf{m}_{1(M-1)}$ will be greater than $T-\xi$, so to build the square matrix we will require an extrapolation.

We construct a matrix $\overline{\mathbf{U}}$ from the above conditions and the equations (16). $\overline{\mathbf{U}}$ is build by the last $n(N+1-(M-1))$ rows of $\mathbf{U}^{2}$ and the $n(M-1)$ rows of $\mathbf{U}$ from row $n+1$. We discard these $n$ first rows due to the continuity condition. The new operator maps $\mathbf{m}_{\varphi}$ to the solution values in the range $\left[\xi+s_{M-1}, 2 \xi+s_{M-1}\right]$, we call it $\overline{\mathbf{m}}$. But this operator is not what we want to find, because the projections of points $\overline{\mathbf{m}}$ do not coincide with the collocation points $\tilde{s}_{j}$, which is clearly shown in Fig. 5 . We use the values $\overline{\mathbf{m}}$ of the solution to find the values $\tilde{\mathbf{m}}$ of the solution in the $\tilde{s}_{j}$ points, and we define (approximately) the operator $\tilde{\mathbf{U}}$.

The easiest way is to perform a linear interpolation of values in $\tilde{\mathbf{m}}$ using the values in $\overline{\mathbf{m}}$. This interpolation is represented by a matrix $\mathbf{M}_{L}$ whose elements are defined in terms of $\tilde{s}_{j}$ and the collocation points $s_{j}+\xi$ and $s_{j}+2 \xi$ in the interval $\left[\xi+s_{M-1}, 2 \xi+s_{M-1}\right]$, that we call $\bar{s}_{j}$. In this way, the operator $\tilde{\mathbf{U}}$ is approximately $\mathbf{M}_{L} \overline{\mathbf{U}}$, and we can approximate the eigenvalues by calculating the eigenvalues of $\mathbf{M}_{L} \overline{\mathbf{U}}$.

One way to measure the accuracy of the approximation is to calculate the error of the trivial eigenvalue 1 , we can see that the error decreases as the number of collocation points increases. However, if we continue increasing the amount of points the error reaches a minimum and it stabilize. We compared the calculated eigenvalues with those obtained by DDE-BIFTOOL [Engelborghs et al., 2001] for particular cases and the results show good agreement. Table 2 shows results obtained for a particular cycle. 
The approximation of the points in $\tilde{\mathbf{m}}$ also can be achieved through barycentric interpolation. This interpolation is defined as

$$
\tilde{\mathbf{m}}_{j}=\frac{\sum_{i=0}^{N} \frac{\eta_{i}}{\tilde{s}_{j}-\bar{s}_{i}} \overline{\mathbf{m}}_{i}}{\sum_{i=0}^{N} \frac{\eta_{i}}{\tilde{s}_{j}-\bar{s}_{i}}}
$$

where $\eta_{i}=(-1)^{i} / 2$ if $i=0$ or $i=N$ and, $\eta_{i}=(-1)^{i}$ if $i \neq 0, i \neq N$.

As in the previous case, we can define a matrix $\mathbf{M}_{B}$, that represents the interpolation in terms of $\tilde{s}_{j}$ and $\bar{s}_{j}$. The operator $\tilde{\mathbf{U}}$ in this case is approximately $\mathbf{M}_{B} \overline{\mathbf{U}}$. The calculation of the eigenvalues of $\mathbf{M}_{B} \overline{\mathbf{U}}$ are compared with those calculated with DDE-BIFTOOL. The comparison shows that the approximation is better than before, for the same number of collocation points $m$ (see Table 2).

Table 2. Eigenvalues of the cycle for $\epsilon=0.1, \tau=7$, and $d=8.3328$.

\begin{tabular}{ccccc}
\hline DDE-BIFTOOL & \multicolumn{2}{c}{ Linear interpolation } & \multicolumn{2}{c}{ Barycentric interpolation } \\
& $m=40$ & $m=100$ & $m=40$ & $m=100$ \\
\hline$-1.2202 \pm 0.8640 i$ & $-1.2203 \pm 0.8614 i$ & $-1.2204 \pm 0.8633 i$ & $-1.2209 \pm 0.8638 i$ & $-1.2204 \pm 0.8638 i$ \\
1.0000 & 0.9893 & 0.9976 & 0.9975 & 0.9992 \\
0.8985 & $0.8898 \pm 0.0022 i$ & 0.8974 & 0.8991 & 0.8984 \\
0.8880 & 0.8883 & 0.8883 & 0.8881 \\
$-0.2232 \pm 0.2750 i$ & $-0.2192 \pm 0.2692 i$ & $-0.2225 \pm 0.2741 i$ & $-0.2228 \pm 0.2752 i$ & $-0.2231 \pm 0.2750 i$ \\
$-0.0680 \pm 0.1979 i$ & $-0.0646 \pm 0.1907 i$ & $-0.0675 \pm 0.1967 i$ & $-0.0683 \pm 0.1977 i$ & $-0.0680 \pm 0.1978 i$ \\
$0.1422 \pm 0.0302 i$ & $0.1332 \pm 0.0291 i$ & $0.1407 \pm 0.0300 i$ & $0.1421 \pm 0.0297 i$ & $0.1420 \pm 0.0301 i$ \\
$-0.0744 \pm 0.0805 i$ & $-0.0682 \pm 0.0725 i$ & $-0.0735 \pm 0.0792 i$ & $-0.0743 \pm 0.0805 i$ & $-0.0743 \pm 0.0804 i$ \\
$-0.0247 \pm 0.0835 i$ & $-0.0208 \pm 0.0730 i$ & $-0.0240 \pm 0.0819 i$ & $-0.0255 \pm 0.0834 i$ & $-0.0246 \pm 0.0834 i$ \\
\hline
\end{tabular}

\section{Near Double Hopf Points}

The knowledge of the cycles and their stability allows us to detect and analyze bifurcations in which they are involved. In this case we focus on the analysis of double Hopf bifurcations. To carry out this analysis we fix $\epsilon$ and find all the cycles and their stability along horizontal lines of the $(d, \tau)$-plane.

\subsection{3 : 4 Resonant Double Hopf}

For $\epsilon=0.139057$, a $3: 4$ resonant double Hopf bifurcation occurs when $d=2.22971$ and $\tau=7.90083$. The pairs of corresponding eigenvalues are $\pm i 1.125888$ and $\pm i 0.844416$. The analysis of the behavior of the cycles near the double Hopf point allows us to detect Neimark-Sacker (NS) bifurcations occurs. It determines the appearance of $2 D$ torus. The observed dynamics corresponds to one of the known as simple cases [Kuznetsov, 1995].

We summarize what happens when the parameters $d$ and $\tau$ vary around the double Hopf point: in region I (see Fig. 6 a)) the equilibrium is stable, going to region II in $H_{1}$ curve the system undergoes a supercritical Hopf bifurcation, and the emerging cycle is stable. It remains stable in region III and there is a new unstable cycle (born in the Hopf bifurcation $H_{2}$ ), which is stabilized by a NS bifurcation (curve $T_{2}$ ). In region IV, both cycles are stable and the $2 D$ torus that arises is unstable. Following, by crossing the curve $T_{1}$ the torus no longer exists. In region $\mathrm{V}$, we find again a stable cycle and an unstable one, the last one disappears due to the Hopf bifurcation $H_{1}$ and the remainder vanishes at $H_{2}$.

The curves $T_{1}$ and $T_{2}$ in $(d, \tau)$-plane are shown in Fig. 6 a), in the same figure you can also see Hopf curves $H_{1}$ and $H_{2}$, and the regions mentioned above. Fig. 6 b) shows the amplitudes of cycles for 

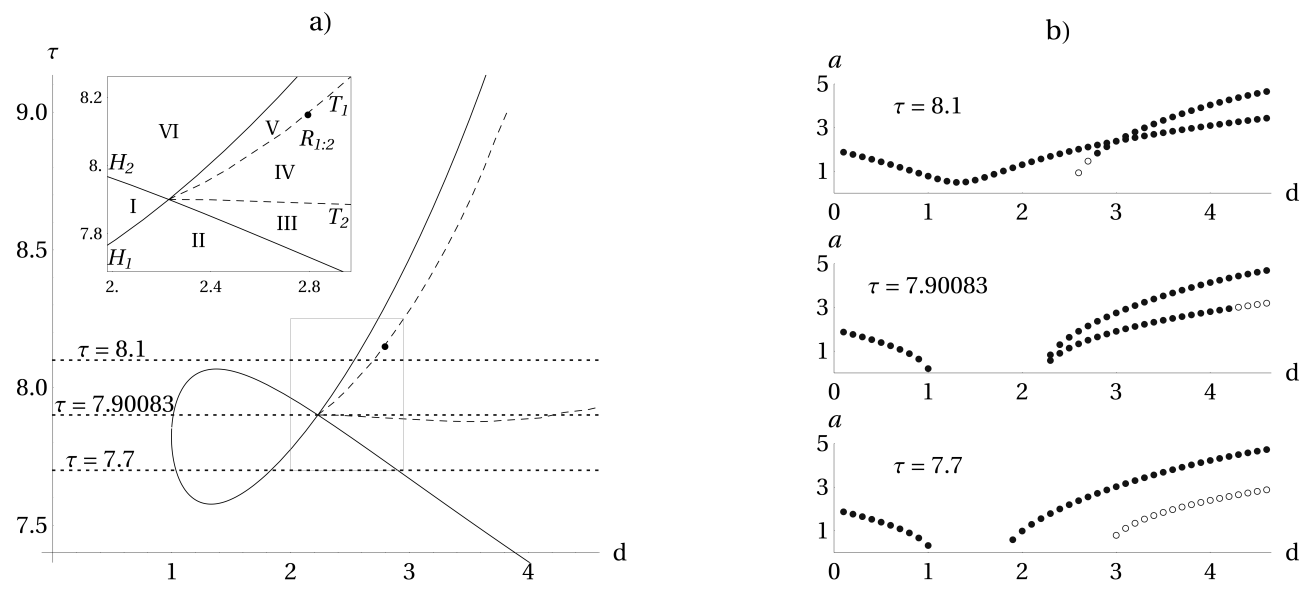

Fig. 6. a) Neighborhood of a double Hopf point, $\epsilon=0.139057$; b) Amplitudes for constant values of $\tau$ shown in a).

various constant values $\tau$, the results coincide with the described dynamics. In this and the next figures the filled dots represent the amplitude of stable oscillations and the unfilled dots represent unstable periodic solutions.

\subsection{Folds Near Double Hopf}

As we mentioned, we may find curves in the space of parameters that correspond to double Hopf points. In some cases they do not involve changes in stability of equilibrium. But they are interesting because of the dynamics of the cycles which is developed. As an example, consider a neighborhood of the double Hopf point obtained for values $\epsilon=0.5, \tau=12.254248$, and $d=1.511726$. The trivial equilibrium does not change its stability in this region and the cycles that arise are unstable.

Fig. $7 \mathrm{~b}$ ) shows the calculation of the amplitude of the existing cycles for $\tau=12$. We observe two branches of unstable cycles arising from Hopf bifurcations. In each branch it is possible to observe a fold bifurcation of cycles, characterized by the presence of an eigenvalue 1 (in addition to the trivial). In one of them the cycle that arises is stable and in the other unstable. The branches are compared with those obtained using DDE-BIFTOOL. Note that using the HAM we found a stable cycle which is independent of local dynamics, it is a deformation of the cycle of the system without delay. Numerical calculations performed with Matlab show that this cycle exist and is stable, but this branch could not be easily found using DDE-BIFTOOL. We do not analyze other bifurcations that could occur near of that double Hopf point.

In Fig 7 a) we depict the situation in a neighborhood of the double Hopf point for the mentioned value of $\epsilon$. There are shown the Hopf curves $H_{1}$ and $H_{2}$ and the fold curves $F_{1}$ and $F_{2}$ calculated with HAM. The dots indicate the fold points obtained using DDE-BIFTOOL.

\subsection{1 : 1 Resonant Double Hopf}

Finally, we consider a double Hopf point with a $1: 1$ resonance. The coordinates in the parameter space are $(\epsilon, d, \tau)=(0.254659,7.85363,0.991860)$ with $\omega_{1,2}=1$.

From double Hopf point NS branches can be found. Together with the Hopf curves they divide the area into four regions, shown in Fig. 8 a). The equilibrium is unstable in the whole area. When crossing the Hopf curve, from region I to II or from I to IV, unstable cycles appear that stabilize in the NS curves, $T_{2}$ and $T_{1}$ respectively. The torus present in the region III is unstable. Moreover, as in the previous case, we observe the presence of a stable cycle that it is related to the system without delay, Fig. 9 a).

A 1:4 resonance appears on the curves $T_{1}$ and $T_{2}$ (see Fig. 8 a)), that is, the corresponding cycle has eigenvalues $e^{ \pm i \pi / 2}$. At these points, the cycle changes its stability when a pair of pure imaginary eigenvalues enters the unit circle. The dynamics near these resonances can be very complex [Kuznetsov, 1995]. Fig. 9 b) 
a)

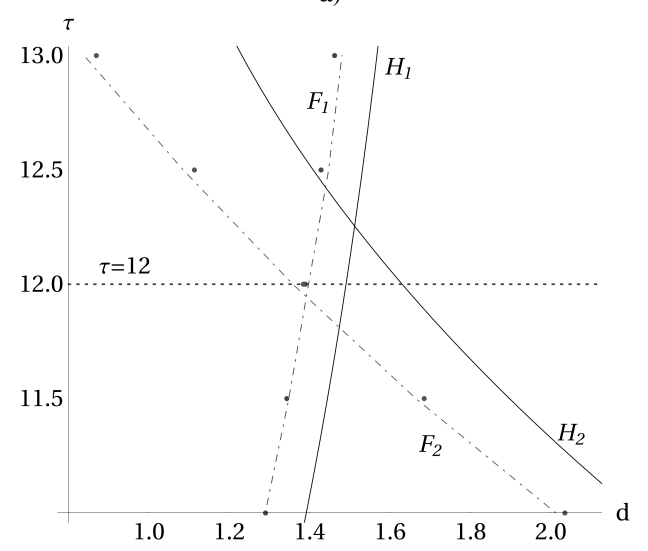

b)

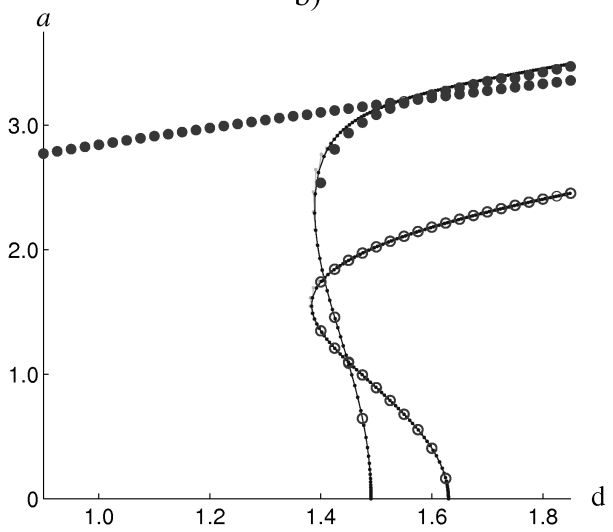

Fig. 7. a) Neighborhood of a double Hopf point, $\epsilon=0.5$; b) Amplitude of cycles for $\tau=12,-$ : DDE-BIFTOOL, ०००: HAM.

a)

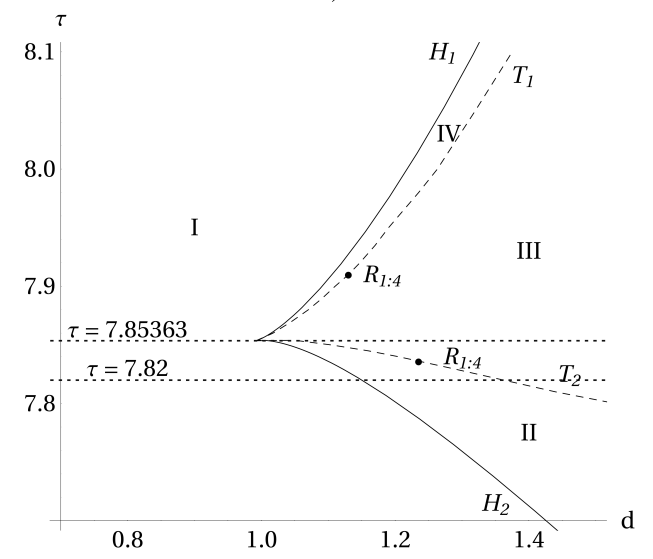

b)

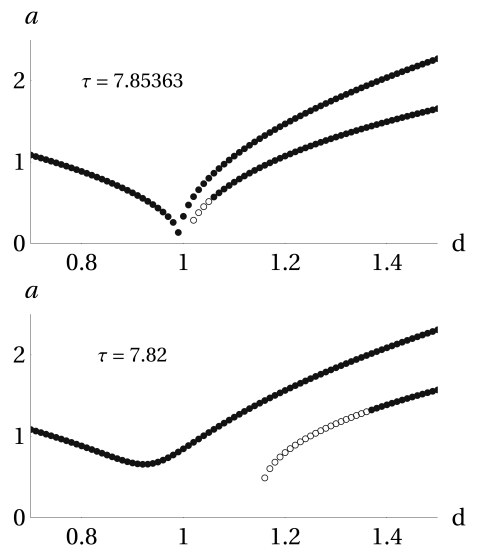

Fig. 8. a) Neighborhood of a $1: 1$ resonant double Hopf point, $\epsilon=0.254659$; b) Amplitudes for constant values of $\tau$ shown in a).
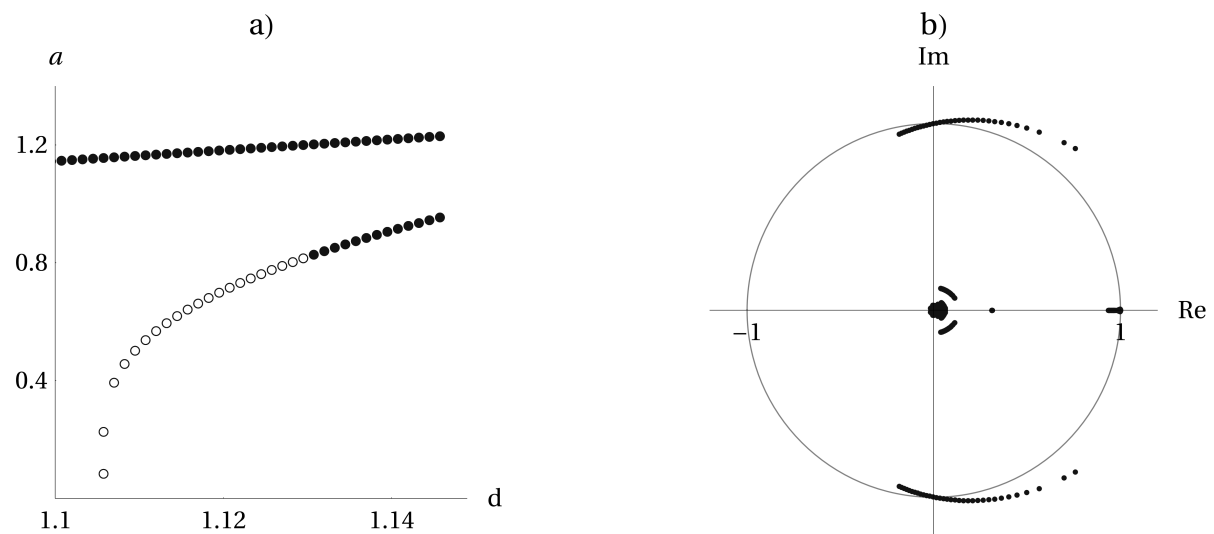

Fig. 9. a) Amplitude of cycles for $\epsilon=0.254659, \tau=7.91$; b) Eigenvalues corresponding to the lower curve in a).

shows this situation to the point $R_{1: 4}$ in $T_{1}$, we can see the eigenvalues in the complex plane corresponding to different values of $d$ for the cycles corresponding to the lower curve in Fig. 9 a). 


\section{Conclusions}

In this work we apply the HAM to find analytical expressions of periodic solutions of a nonlinear delay differential equation. The method allow us to calculate these solutions with great precision, which is observed when the obtained solutions are compared with those obtained by numerical methods. Moreover, the stability study results straightforwardly from the expressions of the solutions. Then, it is possible to survey the parameter space that allow us to characterize bifurcations.

We have shown that the HAM is an appropriate tool for studying nonlinear delay differential equations focussing on the bifurcation analysis and stability of the periodic solutions. Specifically, we have carried out this contribution on a vast region of the parameter space in van der Pol's equation with delayed feedback.

\section{Acknowledgments}

We thank Prof. Blanca Niel for her reading of the manuscript and useful suggestions. Also, we would to thank Dr. Griselda Itovich for valuable discussions about methods in bifurcation analysis of delay differential equations. The work is supported by the Departamento de Matemática of the Universidad Nacional del Sur (UNS) and for the Consejo Nacional de Investigaciones Científicas y Técnicas (CONICET), both from Argentina.

\section{References}

Abbasbandy, S. [2006] "The application of homotopy analysis method to nonlinear equations arising in heat transfer," Physics Letters A 360, 109-113.

Atay, F. M. [1998] "Van der Pol's oscillator under delayed feedback," J. Sound and Vibration 2, 333-339.

Bueler, E. [2004] "Chebyshev collocation for linear, periodic, ordinary and delay differential equations: A posteriori estimates," Cornell University Library URL http://arxiv.org/pdf/math/0409464.

Butcher, E. \& Mann, B. [2009] "Stability analysis and control of linear periodic delayed systems using Chebyshev and temporal finite element methods," Delay Differential Equations, Recent Advances and New Directions (Springer), pp. 93-129.

Engelborghs, K., Luzyanina, T. \& Samaey, G. [2001] "DDE-BIFTOOL v. 2.00: a matlab package for bifurcation analysis of delay differential equations," Technical Report TW 330, Department of Computer Science, K.U. Leuven, Leuven, Belgium, URL http://twr.cs.kuleuven.be/research/software/delay/dde\ -biftool.shtml.

Hale, J. K. \& Lunel, S. M. V. [1993] Introduction to Functional Differential Equations, Applied Mathematical Sciences, Vol. 99 (Springer-Verlag).

He, J.-H. [1999] "Homotopy perturbation technique," Comput. Methods Appl. Mech. Engrg. 178, $257-262$.

Kimiaeifar, A., Saidi, A. R., Bagheri, G. H., Rahimpour, M. \& Domairry, D. G. [2009] "Analytical solution for Van der Pol-Duffing oscillations," Chaos, Solitons and Fractals 42, 2660-2666.

Kuznetsov, Y. A. [1995] Elements of Applied Bifurcation Theory (Springer).

Liao, S. [2004a] "An analytic approximate approach for free oscillations of self-excited systems," International Journal of Non-linear Mechanics 39, 271-280.

Liao, S. [2004b] Beyond Perturbation, Introduction to Homotopy Analysis Method (Chapman \& Hall/CRC).

Liao, S. [2004c] "On the homotopy analysis method for nonlinear problems," Applied Mathematics and Computations 147, 499-513.

Liao, S. [2009] "Notes on the homotopy analysis method: some definitions and theorems," Commun Nonlinear Sci Numer Simulat 14, 983-997.

Liao, S. \& Tan, Y. [2007] "A general approach to obtain series solutions of nonlinear differential equations," Studies in Applied Mathematics 119, 297-354.

Roohi, E., Marzabadi, F. R. \& Farjami, Y. [2008] "Application of the homotopy perturbation method to linear and nonlinear fourth-order boundary value problems," Physica Scripta $\mathbf{7 7 .}$

Sajid, M., Hayat, T. \& Asghar, S. [2007] "Comparison between the HAM and HPM solutions of thin film flows of non-newtonian fluids on a moving belt," Nonlinear Dynamics 50, 27-35. 
Shakeri, F. \& Dehghan, M. [2008] "Solution of delay differential equations via a homotopy perturbation method," Mathematical and Computer Modelling 48, 486-498.

Wei, J. \& Jiang, W. [2005] "Stability and bifurcation analysis in van der Pol's oscillator with delayed feedback," Journal of Sound and Vibration 283, 801-819. 